\title{
BMJ Open Lifetime alcohol use and overall and cause-specific mortality in the European Prospective Investigation into Cancer and nutrition (EPIC) study
}

To cite: Ferrari P, Licaj I, Muller DC, et al. Lifetime alcohol use and overall and cause-specific mortality in the European Prospective Investigation into Cancer and nutrition (EPIC) study. BMJ Open 2014;4:e005245. doi:10.1136/bmjopen-2014005245

- Prepublication history and additional material is available. To view please visit the journal (http://dx.doi. org/10.1136/bmjopen-2014005245).

Received 11 March 2014 Revised 23 April 2014 Accepted 25 April 2014

CrossMark

For numbered affiliations see end of article.

Correspondence to Dr Pietro Ferrari; ferrarip@iarc.fr

\section{ABSTRACT}

Objectives: To investigate the role of factors that modulate the association between alcohol and mortality, and to provide estimates of absolute risk of death.

Design: The European Prospective Investigation into Cancer and nutrition (EPIC).

Setting: 23 centres in 10 countries.

Participants: 380395 men and women, free of cancer, diabetes, heart attack or stroke at enrolment, followed up for 12.6 years on average.

Main outcome measures: 20453 fatal events, of which 2053 alcohol-related cancers (ARC, including cancers of upper aerodigestive tract, liver, colorectal and female breast), 4187 cardiovascular diseases/coronary heart disease (CVD/CHD), 856 violent deaths and injuries. Lifetime alcohol use was assessed at recruitment.

Results: HRs comparing extreme drinkers ( $\geq 30 \mathrm{~g} /$ day in women and $\geq 60 \mathrm{~g} /$ day in men) to moderate drinkers $(0.1-4.9 \mathrm{~g} /$ day) were $1.27(95 \% \mathrm{Cl} 1.13$ to 1.43$)$ in women and 1.53 (1.39 to 1.68) in men. Strong associations were observed for ARC mortality, in men particularly, and for violent deaths and injuries, in men only. No associations were observed for CVD/CHD mortality among drinkers, whereby HRs were higher in never compared to moderate drinkers. Overall mortality seemed to be more strongly related to beer than wine use, particularly in men. The 10-year risks of overall death for women aged 60 years, drinking more than $30 \mathrm{~g} /$ day was $5 \%$ and $7 \%$, for never and current smokers, respectively. Corresponding figures in men consuming more than $60 \mathrm{~g} /$ day were $11 \%$ and $18 \%$, in never and current smokers, respectively. In competing risks analyses, mortality due to CVD/CHD was more

\section{Strengths and limitations of this study}

- This study was based on information on dietary and lifestyle exposure collected in a large prospective investigation of European adults.

- Findings are based on 380395 men and women (among whom 20453 fatal events occurred) for which information on lifetime alcohol use was available, allowing separate consideration of former drinkers from lifetime abstainers.

- Exclusion of study participants reporting a morbid condition at baseline, and sensitivity analyses excluding the first 3 years of follow-up limited the chance that reverse causality affected the findings.

- Although statistical models included many potentially relevant adjustment factors, residual confounding might partially account for the observed associations.

- Average lifetime alcohol consumption was evaluated in this study, whereas it is possible that specific drinking patterns in particular phases of life, as well as the effect of binge drinking or drinking during meals, may also be of particular relevance for mortality.

pronounced than ARC in men, while CVD/CHD and ARC mortality were of similar magnitude in women.

Conclusions: In this large European cohort, alcohol use was positively associated with overall mortality, ARC and violent death and injuries, but marginally to CVD/CHD. Absolute risks of death observed in EPIC suggest that alcohol is an important determinant of total mortality. 


\section{INTRODUCTION}

Alcohol intake has been associated with an increased risk of death from a large list of morbid conditions, including digestive tract conditions, liver cirrhosis, chronic pancreatitis, hypertension, injuries and violence. ${ }^{1-3}$ In contrast, moderate alcohol drinking was suggested to be associated with a reduction in cardiovascular disease (CVD) mortality. ${ }^{4}$ As for cancer, the International Agency for Research on Cancer and the World Cancer Research Fund/American Institute for Cancer Research concluded that alcohol use is associated with an increased risk to develop cancers of the upper aerodigestive tract, liver, colorectal and female breast. ${ }^{6}{ }^{7}$ It has been estimated that alcohol accounted for about 2.7 million annual deaths and $3.8 \%$ of all deaths worldwide, ${ }^{8}{ }^{9}$ but the impact of alcohol on mortality is differential with respect to specific causes of diseases. ${ }^{3}$

Within the European Prospective Investigation into Cancer and nutrition (EPIC), a recent study showed that heavy alcohol use was associated with a higher risk of death from alcohol-related cancer, external causes and 'other causes', while no associations were observed for coronary heart disease and other cardiovascular diseases. ${ }^{10}$

In this study, we further investigated associations between alcohol use and overall and cause-specific mortality. Notably, potential variability of the relationships with respect to smoking habits, the type of alcoholic beverages and country was explored. The cumulative probabilities of death were estimated for overall mortality and, in a competing risks framework, for specific mortality causes with respect to levels of alcohol, separately in men and women. Furthermore, the burden of alcohol use in relation to a broad group of causes of deaths was evaluated by means of overall estimates of rate advancement periods, with respect to two alternative scenarios.

\section{METHODS}

Study population

EPIC is an on-going multicentre study that has been described in detail previously. ${ }^{11}$ From 1992 to 2000, 521448 individuals, aged 25-70 years were recruited in the surroundings of 23 centres in 10 European countries. Most of the participants were recruited from the general population residing in a given geographic area, a town or a province. Exceptions were the cohorts of France (female members of a health insurance scheme for school employees), Utrecht (breast cancer screening attendees), Ragusa (blood donors and their spouses) and Oxford (mainly vegetarian and healthy eaters). Some characteristics of the study population in the different participating countries are reported in table 1 . Study participants provided informed consent and completed questionnaires on their diet, lifestyle and medical history. The study was approved by the relevant ethical review boards of each centre and the International Agency of Research on Cancer in Lyon, France. ${ }^{11}$
Dietary and lifestyle assessment

Diet was assessed at enrolment using validated countryspecific or centre-specific dietary questionnaires designed to capture habitual consumption over the preceding year. Lifetime alcohol consumption was assessed based on self-reported weekly consumption of wine, beer and liquor at ages 20, 30, 40, 50 years in the lifestyle questionnaire. Information on lifetime alcohol consumption was available for approximately $76 \%$ of EPIC participants. ${ }^{12}$ Information on smoking status and duration, physical activity during leisure time, prevalent conditions at baseline, educational attainment, anthropometric measures and reproductive history was obtained using lifestyle questionnaires.

\section{Assessment of causes of death}

Vital status and information on cause and date of death were ascertained using record linkage with cancer registries, boards of health and death registries (Denmark, Italy, the Netherlands, Spain, the UK) or by active follow-up (France Germany, Greece). Data were coded using the 10th revision of the International Statistical Classification of Diseases, Injuries and Causes of Death (ICD-10) where the underlying cause is the official cause of death.

In this work, six different causes of deaths were selected: cardiovascular disease (CVD) (I00-I99 excluding I20-I25) and coronary heart disease (CHD) (I20-I25), alcohol-related cancer (ARC), including colorectal cancer (C18-C20), female breast cancer (C50), upper aerodigestive cancers (UADT, including cancer of the mouth (C01-C10 without C08=salivary gland), larynx (C21), pharynx (C11-C14), oesophagus (C15)), violent deaths and injuries (injury, poisoning and certain other consequences of external causes (S00-T98); deaths due to respiratory diseases (J00-J99); a group for all other causes (including external causes of morbidity and mortality (V01-Y98), unknown causes (R96-R99)).

\section{Statistical analyses}

Participants from Denmark (Aarhus, Copenhagen), France, Germany (Heidelberg, Potsdam), Greece, Italy (Florence, Varese, Ragusa, Turin), the Netherlands (Utrecht), Spain (Asturias, Granada, Murcia, Navarra, San Sebastian) and the UK (Cambridge, Oxford) were eligible for this analysis. We excluded the entire cohorts of Naples (Italy), Bilthoven (the Netherlands), Sweden and Norway because no information on past alcohol use was collected $(\mathrm{n}=118$ 082). Further exclusions concerned participants with incomplete vital status information $(\mathrm{n}=928)$, who had not filled out the dietary or lifestyle questionnaires $(n=11411)$ and participants whose ratio of energy intake to estimated energy requirement $(\mathrm{n}=7592)$, calculated in terms of gender, body weight, height and age, was in the top or bottom $1 \%$ in order to partially reduce the impact of outlier values. ${ }^{13}$ Participants that at recruitment reported cancer $(n=13$ 283), diabetes $(n=11240)$, myocardial infarction 
Table 1 Country-specific and sex-specific number of participants $(\mathrm{N})$, person-years (PY), cause-specific and overall number of deaths

\begin{tabular}{|c|c|c|c|c|c|c|c|c|c|c|c|c|c|c|}
\hline \multirow[b]{2}{*}{ Country } & \multirow[b]{2}{*}{$\mathbf{N}$} & \multirow[b]{2}{*}{ PY } & \multirow[b]{2}{*}{$\mathrm{CHD}^{*}$} & \multirow[b]{2}{*}{ CVD } & \multicolumn{5}{|c|}{ Cancers } & \multirow{2}{*}{$\begin{array}{l}\text { Other } \\
\text { cancers } 1\end{array}$} & \multirow{2}{*}{$\begin{array}{l}\text { Violent and } \\
\text { injuries** }\end{array}$} & \multirow[b]{2}{*}{ Resptt } & \multirow{2}{*}{ 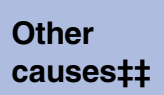 } & \multirow[b]{2}{*}{ Total } \\
\hline & & & & & Breast & UADT‡ & Liver & Colon-rectum & Total§ & & & & & \\
\hline \multicolumn{15}{|l|}{ Women } \\
\hline France & 65127 & 971127 & 45 & 202 & 62 & 8 & 4 & 27 & 101 & 678 & 115 & 73 & 1619 & 2833 \\
\hline Italy & 24956 & 306244 & 26 & 87 & 71 & 6 & 12 & 53 & 142 & 293 & 30 & 12 & 120 & 710 \\
\hline Spain & 23616 & 323027 & 41 & 50 & 51 & 6 & 9 & 47 & 113 & 243 & 46 & 9 & 119 & 621 \\
\hline UK & 50251 & 651640 & 387 & 320 & 160 & 30 & 15 & 127 & 332 & 716 & 85 & 161 & 1091 & 3092 \\
\hline The Netherlands & 14583 & 189531 & 110 & 137 & 58 & 14 & 7 & 78 & 157 & 370 & 20 & 65 & 209 & 1068 \\
\hline Greece & 14391 & 143150 & 139 & 100 & 41 & 2 & 10 & 18 & 71 & 146 & 26 & 27 & 94 & 603 \\
\hline Germany & 27098 & 307380 & 56 & 74 & 64 & 12 & 14 & 44 & 134 & 256 & 35 & 25 & 114 & 694 \\
\hline Denmark & 27773 & 328375 & 84 & 143 & 128 & 23 & 17 & 110 & 278 & 648 & 51 & 116 & 548 & 1868 \\
\hline All & 247795 & 3220474 & 888 & 1113 & 635 & 101 & 88 & 504 & 1328 & 3335 & 408 & 488 & 3848 & 11489 \\
\hline \multicolumn{15}{|l|}{ Men } \\
\hline France & - & - & - & - & - & - & - & - & - & - & - & - & - & - \\
\hline Italy & 13471 & 168992 & 53 & 59 & - & 8 & 10 & 39 & 57 & 240 & 35 & 13 & 131 & 588 \\
\hline Spain & 14089 & 189942 & 136 & 88 & - & 34 & 14 & 62 & 110 & 351 & 81 & 42 & 151 & 959 \\
\hline UK & 20452 & 262720 & 438 & 229 & - & 41 & 11 & 68 & 120 & 567 & 88 & 171 & 1040 & 2653 \\
\hline The Netherlands & - & - & - & - & - & - & - & - & - & - & - & - & - & _- \\
\hline Greece & 9726 & 90989 & 193 & 141 & - & 12 & 20 & 31 & 63 & 279 & 49 & 54 & 99 & 878 \\
\hline Germany & 19743 & 221724 & 167 & 138 & - & 37 & 25 & 65 & 127 & 437 & 84 & 35 & 264 & 1253 \\
\hline Denmark & 24454 & 282622 & 273 & 271 & - & 91 & 31 & 126 & 248 & 838 & 111 & 95 & 794 & 2633 \\
\hline All & 101935 & 1216988 & 1260 & 926 & - & 223 & 111 & 391 & 725 & 2712 & 448 & 410 & 2479 & 8964 \\
\hline
\end{tabular}

${ }^{*} \mathrm{CHD}$, coronary heart disease (I20-I25) deaths.

TCVD, cardiovascular disease (I00-199 except I20-125) deaths.

fUADT deaths from upper aerodigestive cancers (including cancer of the mouth (C01-C10 without C08=salivary gland)), larynx (C21), pharynx (C11-C14), oesophagus (C15)).

$\S$ Total frequency of alcohol-related cancers.

TOther cancers: deaths from all other cancers.

${ }^{\star}$ Violent deaths and injuries, including injury, poisoning and certain other consequences of external causes (S00-T98), and external causes of morbidity and mortality (V01-Y98).

††Resp=respiratory diseases (J00-J99).

$\ddagger \ddagger A l l$ other causes of death. 
or heart disease $(n=5266)$ or stroke $(n=3246)$ were excluded from the analyses ( $\mathrm{n}=30665$ in total).

Cox proportional hazard models were used to compute mortality HR, and 95\% CIs, for categories of average lifetime alcohol use; never drinkers, $1-4.9 \mathrm{~g} /$ day (reference category), 5-14.9, 15-29.9, 30-59.9, $\geq 60 \mathrm{~g}$ /day. In women, the last two alcohol categories were collapsed into a $\geq 30 \mathrm{~g} /$ day group. Time in the study up to death, loss or end of follow-up, whichever came first was the primary time variable. The Breslow method was adopted for handling ties. Models were stratified by centre to control for differences in questionnaire design, follow-up procedures and other centre-specific effects. ${ }^{13}$ Systematic adjustments were undertaken for age at recruitment, body mass index and height (continuous), an indicator for participants who quitted alcohol drinking, time since alcohol quitting (continuous), smoking (never, current with 1-15 cigarettes/day, current with more than 15 cigarettes/day, former smoker that quitted less than 10 years before recruitment, former smoker that quitted more than 10 years before recruitment, current smoker of other than cigarettes, unknown $(\mathrm{n}=8819)$ ), duration of smoking (continuous), age at start smoking (less than 19 years, more than 19 years, unknown $(n=39041))$, educational attainment (five categories of level of schooling: none, primary, technical or degree or more, unknown $(\mathrm{n}=14223))$ as a proxy variable for socioeconomic status, physical activity (inactive, moderately inactive, moderately active, active, unknown $(\mathrm{n}=328))$ and energy intake (continuous). In women the models were further adjusted for menopausal status (dichotomised as natural postmenopausal or surgical vs premenopausal or perimenopausal, as assessed at baseline), ever use of replacement hormones, and number of full-term pregnancies (nulliparous, one or two children, more than three, unknown $(n=6482)$. Indicator variables specific to some of the confounding factors were used to model missing values, after checking that the parameters associated with these indicators were not statistically significantly associated with risk of death.

Models for overall and cause-specific mortality were fitted, separately for men and women. An overall test of significance of HRs related to alcohol use was determined by computing $p$ values $\left(p_{\text {Wald }}\right.$ ) for Wald test statistics compared with a $\chi^{2}$ distribution with degrees of freedom equal to the number of alcohol categories minus one. The proportional hazards assumption in the Cox model was satisfied and evaluated via inclusion into the disease model of interaction terms between lifetime alcohol and follow-up time. To reduce the chance of reverse causality, sensitivity analyses were run excluding the first 3 years of follow-up. As results were not different from those using the entire cohort, they were not shown. Analyses excluding former drinkers $4 \%$ and $5 \%$ of the study populations, in men and women, respectively) provided very similar results (results not shown).
Evaluating heterogeneity

Effect modification in the relation between alcohol and mortality by, in turn, smoking status (never, ever), smoking status (never and current smokers) and recruitment country was assessed. Models with main effects and interaction terms were fitted and compared with models with main effects only. The difference in log-likelihood (likelihood ratio test statistics) was compared to a $\chi^{2}$ distribution with degrees of freedom equal to the number of interaction terms. HRs for alcohol categories across levels of interacting variables were computed as linear combinations of main effects and interactions. Associations with wine and beer use (each grouped as never, $0.1-2.9 \mathrm{~g} /$ day (reference), $3-9.9, \quad 10-19.9, \quad 20-39.9, \quad \geq 40 \mathrm{~g} /$ day, $\quad \geq 20 \mathrm{~g} /$ day in women) and total mortality were assessed in mutually adjusted models. The difference of association for wine and beer use in relation to overall mortality was assessed by inspecting the significance of the parameter related to the arithmetic difference of wine and beer use (expressed on the log-scale plus 1 to deal with abstainers) in a model that also included their arithmetic sum. When assessing the association for wine (beer) intake, analyses were restricted to moderate lifetime drinkers of beer (wine) and spirits (below $3 \mathrm{~g} /$ day).

Flexible parametric survival models ${ }^{14}$ with age as the time scale were used to evaluate whether the association between alcohol intake and mortality rate varied by attained age. The origin of the time scale was set to 30 years as the hazard of death is essentially zero prior to that age. The baseline cumulative hazard was modelled using restricted cubic splines with three internal knots placed at evenly spaced centiles of the uncensored log-survival times in order to ensure that an equivalent number of deaths occurred between each knot. ${ }^{15}$ Interactions between alcohol intake and the time scale were modelled using restricted cubic splines with one knot placed at the median of uncensored log-survival times. HRs and differences in survival functions were calculated from the fitted models and plotted against attained age, along with CIs calculated based on $\delta$ method variance estimates.

Possible departures from linearity in the association between average lifetime alcohol use and total mortality were assessed using fractional polynomials, ${ }^{16}$ a subset of generalised linear models in which various powers $(-2$, $-1,-0.5,0,0.5,1,2,3)$ of the covariate(s) of interest are entered into the linear predictor. Fractional polynomials of order two were consistently used in this work for lifetime alcohol use. ${ }^{17}$ Non-linearity was tested comparing the difference in log-likelihood of a model with the fractional polynomials with a model with a linear term only to a $\chi^{2}$ distribution with three degrees of freedom. ${ }^{16}$

\section{Absolute risks}

An extension of the Cox proportional hazards model was employed to fit cause-specific associations between 
lifetime alcohol use and cause-specific mortality in a competing risks framework. ${ }^{18}$ An augmented data set was created where the initial data set is replicated a number of times equal to the different causes of death. In each replicated data set, competing causes of death were set to censored observations and the analyses were stratified by the event type. ${ }^{19}$ The relationship between each confounder variables and cause-specific mortality was assumed homogeneous across causes of death. In this way competing risks were accounted for, and cumulative cause-specific and overall mortality curves were estimated for heavy (greater than 30 and $60 \mathrm{~g} /$ day, in women and men, respectively) and moderate (0.1-4.9 g/day) drinkers, separately for never and current smokers. $^{20}$ Cumulative mortality curves were obtained for participants aged 60 years, using mean values for continuous confounding factors and average frequencies for categorical confounders.

\section{Quantifying the alcohol burden}

The burden of alcohol on mortality was quantified with estimates of the rate advancement period (RAP), ${ }^{21}$ according to two scenarios with threshold levels equal to 5 and $15 \mathrm{~g} /$ day. For overall and cause-specific risk of death, RAP were computed, dividing the $\log (\mathrm{HR})$ estimate comparing alcohol users above and the threshold with alcohol drinkers between $0.1 \mathrm{~g}$ /day and the threshold, by the log of the parameter associated with age. Never alcohol users were not included in the estimation. Associated 95\% CIs were also determined. RAP estimates express the impact of a given exposure on the risk of death, by determining the time (in years) by which the risk of death is anticipated for exposed study participants compared with non-exposed.

Statistical tests were two-sided, and $\mathrm{p}$ values $<0.05$ were considered statistically significant. All analyses were performed using SAS V.9.2 $2^{22}$ and Stata V.12.1. ${ }^{23}$

\section{RESULTS}

\section{Baseline characteristics}

The current analysis was based on 247795 female and 101935 male study participants. The median age at enrolment was 52 years for women and 53 years for men. Study participants were followed on average 12.6 years, accumulating 4800585 person-years, during which a total of 20453 fatal events were recorded (table 1).

Drinking patterns differed substantially between men and women (table 2). In women, $10 \%(n=25146)$ of participants were lifetime never drinkers, while $45 \%$ $(\mathrm{n}=112281)$ and $2 \% \quad(\mathrm{n}=6042)$ were moderate $(0.1-$ $4.9 \mathrm{~g} /$ day) and heavy users (>30 g/day), respectively. Conversely, only $1.5 \%(\mathrm{n}=1600)$ of men reported having never consumed alcohol, $14 \%(n=14287)$ were moderate drinkers, while $29 \% \quad(n=29124)$ were heavy or extreme drinkers (30-59.9 and $\geq 60 \mathrm{~g} /$ day $)$. Furthermore, the vast majority of women who were regular drinkers (total alcohol intake $\geq 10 \mathrm{~g} /$ day) drank predominantly wine $(91 \%)$, rather than beer $(9 \%)$, while regular drinkers in men drank beer (46\%) and wine $(54 \%)$ in similar proportions.

Compared to never and moderate drinkers, women with higher alcohol use had higher levels of education and physical activity, and were more likely to be current smokers or premenopausal/perimenopausal. Never alcohol users were less likely to have used hormonal replacement therapy than alcohol drinkers. In men, the trends were somewhat less apparent. Heavy and extreme alcohol users ( $\geq 30 \mathrm{~g} /$ day) were more often current smokers, attained lower educational level and had higher energy intake levels, compared with moderate drinkers. Never drinkers were physically less active than alcohol drinkers.

\section{Lifetime alcohol and total mortality}

Lifetime average alcohol use was strongly associated with total mortality, in that never and heavy drinkers ( $\geq 30 \mathrm{~g} /$ day) had notably higher mortality rates than did light to moderate drinkers (0.1-4.9 g/day), a pattern that was consistently apparent among female and male study participants (figure 1). The HR comparing never and heavy drinkers with moderate drinkers in women was 1.26 (95\% CI 1.18 to 1.35 ) and 1.27 (1.13 to 1.43$)$, respectively. The corresponding HRs among men were 1.29 (1.10 to 1.51 ) for never drinkers, 1.15 (1.06 to 1.24) for heavy drinkers and 1.53 (1.39 to 1.68) for extreme drinkers ( $\geq 60 \mathrm{~g} /$ day $)$.

\section{Lifetime alcohol and cause-specific mortality}

In men, extreme alcohol use was associated with mortality due to ARCs $\left(\mathrm{HR}_{\geq 60}\right.$ vs ref=2.62 1.90 to 3.62), other cancers (1.34 1.13 to 1.59$)$, violent deaths and injuries (1.93 1.27 to 2.91 ) and other causes (1.98 1.67 to 2.34). With the exception of the category for never drinkers, alcohol intake was not associated with CVD or CHD mortality, in women and men. Among women, heavy drinkers displayed $\mathrm{HR}_{\geq 30}$ vs ref equal to 1.49 (1.07, 2.06) for ARCs. Respiratory diseases were not associated with lifetime alcohol in women, while results were suggestive of an increased risk in extreme alcohol users compared with moderate users in men (see online supplementary figure S1). Dose-response relationships evaluated with fractional polynomials are displayed in online supplementary figures S2 and S3, for women and men, respectively.

\section{Evaluating heterogeneity}

In both sexes, alcohol-related HRs for overall mortality were of similar magnitude in never and current smokers (table 3). Analyses conducted by smoking intensity (never vs heavy smokers, ie, more than 15 cigarettes/day) produced very similar evidence (results not shown). Cause-specific analyses showed mostly homogeneous alcohol-related HRs by smoking status (results not shown). In women, beer use was more strongly related than wine 
Table 2 Characteristics of the study population at recruitment, according to amount and type of alcohol intake (g/day) in the EPIC study*

\begin{tabular}{|c|c|c|c|c|c|c|c|c|c|c|}
\hline \multirow[b]{2}{*}{ Characteristics } & \multirow[b]{2}{*}{ Unit } & \multirow[b]{2}{*}{ Never drinkers } & \multicolumn{5}{|c|}{ Lifetime drinkers } & \multirow[b]{2}{*}{ Totalł } & \multirow{2}{*}{$\begin{array}{l}\text { Wine } \\
\text { consumers } \$\end{array}$} & \multirow{2}{*}{$\begin{array}{l}\text { Beer } \\
\text { consumers } \neq\end{array}$} \\
\hline & & & $0.1-4.9$ & $5-14.9$ & 15-29.9 & 30-59.9† & $>60 \dagger$ & & & \\
\hline \multicolumn{11}{|l|}{ Women } \\
\hline Number of participants & $\mathrm{n}$ & 25146 & 112281 & 77147 & 27179 & 6042 & - & 247795 & 85965 & 8748 \\
\hline Person-years & - & 330854 & 1460315 & 998547 & 352220 & 78538 & - & 3220474 & 1124546 & 110761 \\
\hline Age at recruitment & Years & $52(9)$ & $52(10)$ & $51(10)$ & $49(11)$ & $47(11)$ & - & $51(38-63)$ & $52(9)$ & $46(12)$ \\
\hline Lifetime alcohol intake & g/day & $0(-)$ & $2(2)$ & $9(3)$ & $20(4)$ & $43(21)$ & - & $7(0-17)$ & $12(9)$ & $11(9)$ \\
\hline Educational attainment & $\%$ & 14 & 22 & 27 & 33 & 37 & - & 25 & 28 & 28 \\
\hline Current smokers & $\%$ & 13 & 14 & 18 & 24 & 31 & - & 17 & 17 & 28 \\
\hline Body mass index & $\mathrm{kg} / \mathrm{m}^{2}$ & $27(5)$ & $25(5)$ & $25(4)$ & $24(4)$ & $24(4)$ & - & $25(20-31)$ & $24(4)$ & $25(4)$ \\
\hline Height & $\mathrm{cm}$ & $158(6)$ & $161(6)$ & $162(6)$ & $163(7)$ & $164(6)$ & - & $162(153-170)$ & $162(6)$ & $163(7)$ \\
\hline (Moderately) active & $\%$ & 26 & 39 & 44 & 44 & 43 & - & 40 & 42 & 42 \\
\hline Ever use of $\mathrm{HRT}^{\star \star}$ & $\%$ & 16 & 25 & 29 & 28 & 25 & - & 25 & 50 & 34 \\
\hline Postmenopausal status†† & $\%$ & 50 & 49 & 49 & 44 & 38 & - & 48 & 29 & 20 \\
\hline Energy intake & kcal/day & $1848(537)$ & $1943(537)$ & $2015(536)$ & $2090(552)$ & $2195(602)$ & - & $1978(542)$ & $2046(544)$ & $1976(545)$ \\
\hline \multicolumn{11}{|l|}{ Men } \\
\hline Number of participants & $\mathrm{n}$ & 1600 & 14287 & 28875 & 28049 & 20788 & 8336 & 101935 & 26137 & 22136 \\
\hline Person-years & - & 19114 & 171739 & 345899 & 333784 & 247612 & 98841 & 1216989 & 317937 & 259934 \\
\hline Age at recruitment & Years & $53(11)$ & $53(11)$ & $53(9)$ & $52(9)$ & $52(9)$ & $52(9)$ & $53(41-64)$ & $53(9)$ & $52(10)$ \\
\hline Lifetime alcohol intake & g/day & $0(-)$ & $2(2)$ & $10(3)$ & $22(4)$ & $42(8)$ & $94(45)$ & $25(3-45)$ & $30(27)$ & $22(25)$ \\
\hline Educational attainmentๆ & $\%$ & 21 & 30 & 31 & 32 & 26 & 14 & 29 & 22 & 27 \\
\hline Current smokers & $\%$ & 28 & 22 & 25 & 30 & 36 & 49 & 30 & 31 & 33 \\
\hline Body mass index & $\mathrm{kg} / \mathrm{m}^{2}$ & $27(4)$ & $26(4)$ & $26(3)$ & $27(3)$ & $27(4)$ & $28(4)$ & $27(22-31)$ & $27(4)$ & $27(4)$ \\
\hline Height & $\mathrm{cm}$ & $171(7)$ & $174(7)$ & $175(7)$ & $175(7)$ & $174(7)$ & $172(7)$ & $174(165-183)$ & $172(7)$ & $175(7)$ \\
\hline (Moderately) active & $\%$ & 42 & 46 & 50 & 52 & 52 & 50 & 50 & 48 & 52 \\
\hline Energy intake & kcal/day & $2284(675)$ & 2267 (650) & 2315 (618) & 2417 (622) & $2569(646)$ & $2789(716)$ & $2427(656)$ & $2487(652)$ & $2369(651)$ \\
\hline
\end{tabular}

${ }^{*}$ Means \pm SDs are presented for continuous variables, frequencies for categorical variables.

†ln women the last alcohol category is $\geq 30 \mathrm{~g} /$ day.

For continuous variables (with exception of energy intake), mean (10-90th centile) values are reported.

$\S S t u d y$ participants consuming more than $10 \mathrm{~g} /$ day of wine (or beer), and consuming less than $3 \mathrm{~g} /$ day of beer (or wine).

IParticipants with a university degree or more.

*HRT=hormonal replacement therapy.

††Postmenopausal women plus women who underwent an ovariectomy. 

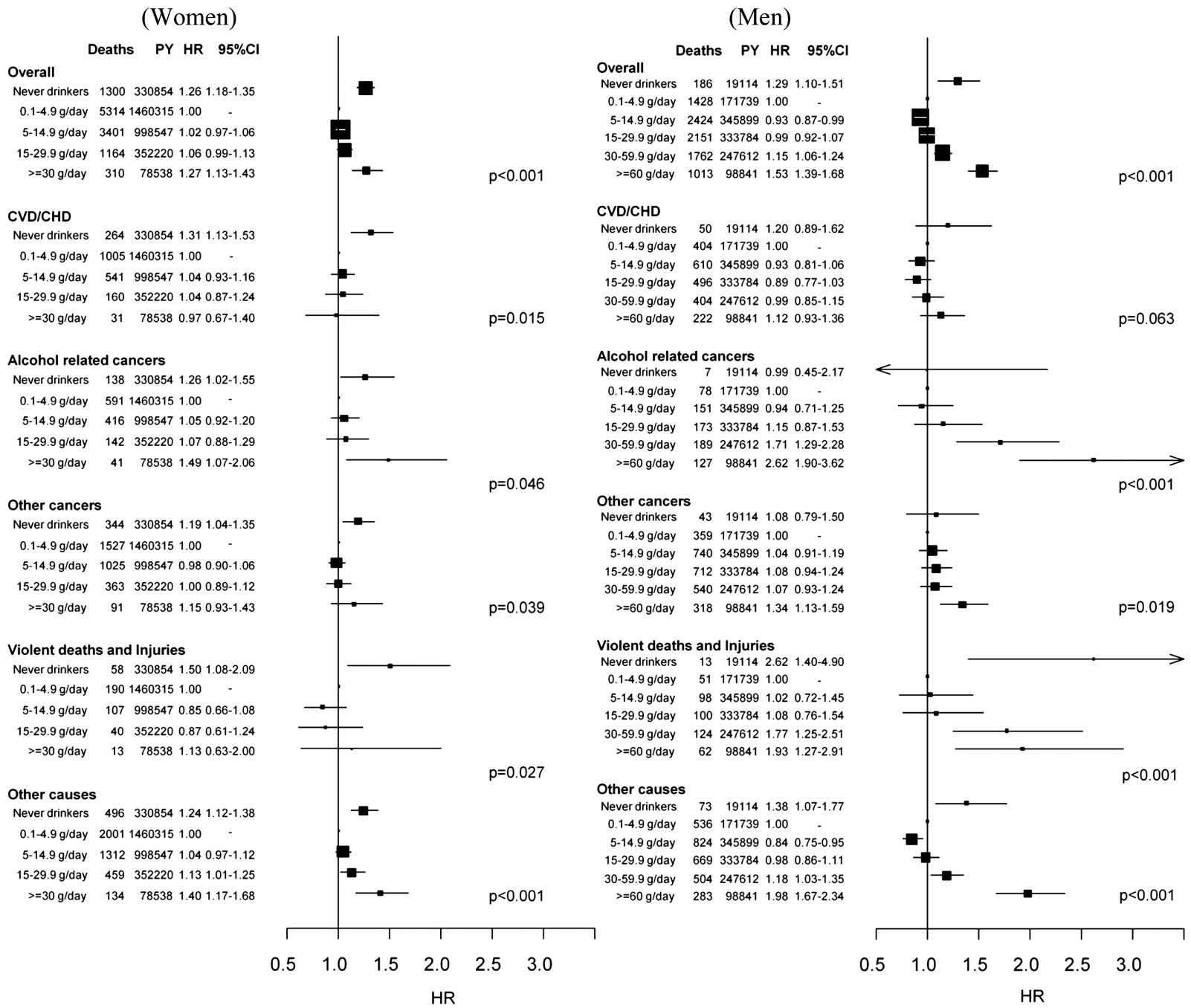

Figure 1 Number of deaths, person-years (PY) and multivariable HRs (Models were stratified by centre. Systematic adjustment was undertaken for age at recruitment, body mass index and height, former drinking, time since alcohol quitting, smoking status, duration of smoking, age at start smoking, educational attainment and energy intake. In women adjustment was undertaken for menopausal status, ever use of replacement hormones and number of full-term pregnancies.) with $95 \%$ Cls and $p$ value of the Wald test for statistical significance for overall and cause-specific mortality by categories of lifetime alcohol use, in women and men.

to overall mortality for amounts greater than $3 \mathrm{~g} /$ day compared with the reference category $(0.1-2.9 \mathrm{~g} /$ day $)$. Lifetime never wine and beer users displayed higher risks than moderate drinkers. The associations between lifetime alcohol and overall risk of mortality were differential across country of origin in men ( $p_{\text {heterogeneity }}=0.012$ ) but not in women ( $p_{\text {heterogeneity }}=0.511$ ), as reported in online supplementary figures S4 and S5, with stronger relationships observed in Northern European countries compared with Southern European countries.

The $\mathrm{HR}_{\geq 60}$ vs ref for men was more pronounced at earlier ages, and were close to one as attained age approached 90 years ( plikelihood-ratio $_{\text {for age-varying vs age }}$ invariant parameterisation $=0.003$ ); however, extreme male drinkers exhibited lower cumulative survival probability than the reference group throughout the lifespan (see online supplementary figure $\mathrm{S6}$ ). No such age-varying association was apparent for women $\left(p_{\text {likelihood-ratio }}=0.80\right)$.

\section{Absolute risks}

The 10-year risk of death at the age of 60 years for heavy drinkers was $5 \%$ and $7 \%$ in women $(\geq 30 \mathrm{~g} /$ day $)$, and $11 \%$ and $18 \%$ in men $(\geq 60 \mathrm{~g} /$ day $)$, for never and current smokers, respectively (figure 2). Corresponding figures in moderate drinkers $(0.1-4.9 \mathrm{~g} /$ day $)$ were $3 \%$ and $4 \%$ in women, and $5 \%$ and $8 \%$ in men. Based on a competing risks analysis, it was estimated that, at the age of 60 years, a female lifetime heavy alcohol drinker and smoker had a 10-year risk of death of $1 \%$ for ARC, $1.2 \%$ for CVD/CHD and $0.2 \%$ for violent death and injuries, as displayed in figure 3. Corresponding figures for males $(\geq 60 \mathrm{~g} /$ day $)$ were $2.2 \%$ (ARC), $5 \%$ (CVD/CHD) and $1 \%$ (violent death and injuries). Risks for moderate drinkers for ARC, CVD/CHD and violent death and injuries were $0.8 \%, 1.2 \%, 0.2 \%$ and $0.9 \%, 4 \%, 0.3 \%$, in women and men, respectively. Consistently lower risks were observed for never smoker individuals, with 


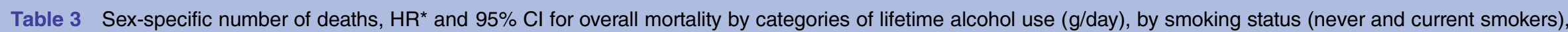
and type of alcoholic beverage

\begin{tabular}{|c|c|c|c|c|c|c|c|c|c|c|c|c|c|c|c|}
\hline \multirow[b]{3}{*}{ Overall } & \multicolumn{6}{|l|}{ Women } & \multirow[b]{3}{*}{$p_{\text {heterog }} \neq$} & \multirow[b]{3}{*}{ Overall } & \multicolumn{6}{|l|}{ Men } & \multirow[b]{3}{*}{$p_{\text {heterog }} \ddagger$} \\
\hline & \multicolumn{3}{|c|}{ Never smokers } & \multicolumn{3}{|c|}{ Current smokers } & & & \multicolumn{3}{|c|}{ Never smokers } & \multicolumn{3}{|c|}{ Current smokers } & \\
\hline & Deaths & $\mathrm{HRT}$ & $(95 \% \mathrm{Cl})$ & Deaths & $\mathrm{HR} \dagger$ & $(95 \% \mathrm{Cl})$ & & & Deaths & $\mathrm{HR} \dagger$ & $(95 \% \mathrm{Cl})$ & Deaths & $\mathrm{HR} \dagger$ & $(95 \% \mathrm{Cl})$ & \\
\hline Never & 1009 & 1.34 & (1.24 to 1.45$)$ & 154 & 1.72 & (1.32 to 2.23$)$ & & Never & 84 & 1.50 & (1.19 to 1.21$)$ & 58 & 2.09 & (1.26 to 3.47$)$ & \\
\hline $0.1-4.9$ & 3046 & 1 & Ref & 1021 & 1.53 & (1.23 to 1.90$)$ & & $0.1-4.9$ & 457 & 1 & Ref & 367 & 1.62 & (1.04 to 2.53$)$ & \\
\hline $5-14.9$ & 1550 & 1.04 & (0.98 to 1.11$)$ & 874 & 1.51 & (1.21 to 1.88$)$ & & $5-14.9$ & 538 & 0.93 & (0.82 to 1.06$)$ & 799 & 1.45 & (0.93 to 2.25$)$ & \\
\hline $15-29.9$ & 397 & 1.04 & (0.94 to 1.16$)$ & 435 & 1.74 & (1.38 to 2.19 ) & & $15-29.9$ & 369 & 1.00 & (0.87 to 1.16$)$ & 927 & 1.66 & (1.06 to 2.58$)$ & \\
\hline$\geq 30$ & 82 & 1.29 & (1.03 to 1.61$)$ & 140 & 2.08 & (1.59 to 2.73 ) & & $30-59.9$ & 254 & 1.22 & (1.23 to 1.43$)$ & 857 & 1.83 & (1.17 to 2.84$)$ & \\
\hline \multirow[t]{4}{*}{$p_{\text {Wald }} \S$} & & & $<0.001$ & & & $<0.001$ & 0.150 & $\geq 60$ & 107 & 1.56 & (1.25 to 1.95$)$ & 590 & 2.43 & (1.55 to 3.80 ) & \\
\hline & & & & & & & & $p_{\text {Wald }} \S$ & & & $<0.001$ & & & $<0.001$ & 0.864 \\
\hline & \multicolumn{3}{|c|}{ Wine use } & \multicolumn{3}{|c|}{ Beer use } & & & \multicolumn{3}{|c|}{ Wine use } & \multicolumn{3}{|c|}{ Beer use } & \\
\hline & Deaths & HRף & $(95 \% \mathrm{Cl})$ & Deaths & HRๆ & (95\% Cl) & P difference $^{\star \star}$ & & Deaths & HRף & (95\% CI) & Deaths & HRף & (95\% Cl) & P difference $^{\star \star}$ \\
\hline Never & 2156 & 1.15 & (1.09 to 1.22$)$ & 5041 & 1.06 & (1.02 to 1.12 ) & & Never & 1064 & 1.21 & (1.12 to 1.30$)$ & 975 & 1.07 & (0.98 to 1.16$)$ & \\
\hline $0.1-2.9$ & 5109 & 1 & Ref & 5477 & 1 & Ref & & $0.1-2.9$ & 3266 & 1 & Ref & 2959 & 1 & Ref & \\
\hline $3-9.9$ & 2813 & 0.96 & (0.92 to 1.01$)$ & 787 & 1.15 & (1.07 to 1.24$)$ & & $3-9.9$ & 2139 & 0.92 & (0.87 to 0.97$)$ & 2486 & 1.04 & (0.98 to 1.10$)$ & \\
\hline $10-19.9$ & 1057 & 1.00 & (0.93 to 1.07 ) & 147 & 1.50 & (1.27 to 1.77 ) & & $10-19.9$ & 1040 & 0.96 & (0.89 to 1.03 ) & 1248 & 1.12 & (1.04 to 1.20$)$ & \\
\hline$\geq 20$ & 354 & 1.14 & (1.02 to 1.27$)$ & 37 & 1.47 & (1.06 to 2.04 ) & & 20-39.9 & 814 & 1.03 & (0.95 to 1.13$)$ & 877 & 1.41 & (1.30 to 1.54$)$ & \\
\hline $\mathrm{p}_{\text {Wald }} \S$ & & & $<0.001$ & & & $<0.001$ & $<0.001$ & $\begin{array}{l}\geq 40 \\
p_{\text {Wald} \S}\end{array}$ & 641 & 1.22 & $\begin{array}{l}(1.10 \text { to } 1.35) \\
<0.001\end{array}$ & 419 & 1.86 & $\begin{array}{l}\text { (1.66 to } 2.09) \\
<0.001\end{array}$ & $<0.001$ \\
\hline
\end{tabular}

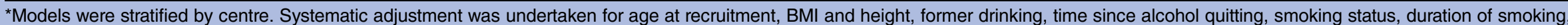
age at start smoking, educational attainment and energy intake. In women adjustment was undertaken for menopausal status, ever use of replacement hormones and number of full-term pregnancies.

†Models included interaction terms between lifetime alcohol use and a smoking indicator ( $0=$ never smokers; $1=$ current smokers), keeping the reference category the group of moderate alcoho users (0.1-4.9 g/day) among never smokers, whereas former smokers and participants with unknown smoking status were excluded.

¥Pheterogeneity: difference in HRs assessed comparing the log-likelihood of models with and without interaction terms between alcohol and smoking status to a four and five degrees of freedom (dof) $\chi^{2}$ distribution, in women and men, respectively.

$\S p_{\text {Wald: }}$ determined using a Wald test for contrasts according to a $\chi^{2}$ distribution with four and five degrees of freedom, in women and men, respectively

१Models on wine and beer uses were mutually adjusted, and also included spirits/liquors use.

${ }^{* *} p_{\text {difference }}$ expresses the difference of associations between wine and beer use, determined evaluating the significance of the parameter estimate $\gamma_{2}$ in a model that included, other than the list of confounders, the terms $\gamma_{1}\left(X_{1}+X_{2}\right) / 2+\gamma_{2}\left(X_{1}-X_{2}\right) / 2$, with $X_{1}=\log ($ wine use +1$)$ and $X_{2}=\log ($ beer use +1$)$ 

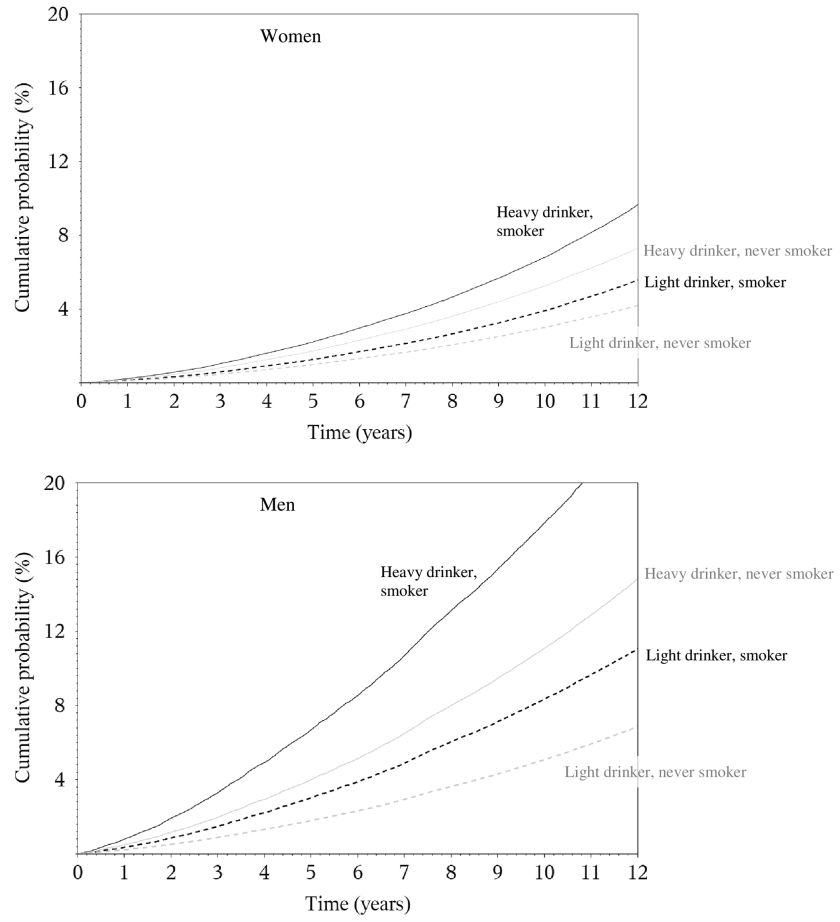

Figure 2 Sex-specific plots displaying cumulative probabilities of death due to overall mortality, for heavy (=30 g/day in women and $=60 \mathrm{~g} /$ day in men, continuous line) and moderate lifetime use (0.1-4.9 g/day) (dotted line), in smokers (black line) and never smokers (grey line), for study participants aged 60 years.

estimates equal to $0.5 \%, 0.7 \%$ and $0.1 \%$ in women, and $1 \%, 2.1 \%$ and $0.3 \%$ in men.

\section{Rate advancement period}

The impact of lifetime alcohol on overall and causespecific mortality was estimated with RAP values (table 4). In women, RAP for overall mortality were equal to 0.36 years ( $95 \%$ CI -0.05 to 0.76 ) and 0.83 (0.26 to 1.39 ), for the 5 and $15 \mathrm{~g} /$ day scenario, respectively. In men, RAP values were equal to 0.15 ( -0.48 to 0.76$)$ and 1.42 (0.96 to 1.89 ), for 5 and $15 \mathrm{~g} /$ day, respectively. RAP values were sizeable for mortality due to ARC (5.03: 3.07, 7.00) and violent death and injuries (11.83: 3.92, 18.17) in the second scenario.

\section{DISCUSSION}

In this large European prospective study, the association between alcohol use and overall and cause-specific risk of death was evaluated in eight European populations. When accounting for potential confounding factors, average lifetime alcohol use was strongly associated with overall mortality, whereas lifetime never alcohol users consistently displayed a higher risk of death compared with moderate drinkers. These results are in agreement with a recent evaluation of alcohol and cause-specific mortality in EPIC. ${ }^{10}$ With respect to this recent study, ${ }^{10}$ further analyses were conducted to deeply investigate the role of factors that modulate the association between alcohol use and the risk of death, notably smoking and types of alcoholic beverage. Estimates of 10-year risk of death in relation to levels of alcohol use were provided.

This study has several strengths. It was conducted using a large prospective cohort, where dietary and lifestyle exposure information were collected on diseasefree individuals. Information on lifetime alcohol use was available on $76 \%$ of the cohort, allowing separate consideration of former drinkers and lifetime abstainers. Further, exclusion of study participants reporting a morbid condition at baseline, and sensitivity analyses excluding the first 3 years of follow-up suggest that reverse causality is unlikely to have affected the results. One potential weakness of this study is that, although statistical models included many potentially relevant adjustment factors, residual confounding might partially account for the observed associations. In addition, average lifetime alcohol consumption was used throughout this study, whereby it is possible that specific drinking patterns in particular phases of life,${ }^{10}$ as well as the effect of binge drinking or drinking during meals may be of particular relevance for mortality.

A recent Russian study found a strong relationship between vodka and risk of death. ${ }^{24}$ While an apparent J-shaped relationship between alcohol use and mortality has been reported,${ }^{25} 26$ the interpretation of this pattern is the subject of some controversy. It has been suggested that alcohol abstinence does not truly entail greater risk of death than moderate use, and that misclassification of alcohol quantity and lack of accuracy in reporting prevalent morbid conditions at baseline in the group of never drinkers ${ }^{27}$ could explain the excess risks observed. This reasoning motivated our choice of considering moderate alcohol drinkers, as the reference category throughout this work. Moreover, residual and unmeasured confounding are plausible drivers of the association. ${ }^{28}$ These suggestions are supported by our findings that never drinkers are at increased risk of death due to violence and injury. This implausible association casts considerable doubt on the veracity of the apparent increased risk of death among never drinkers.

The overall mortality HR for men with extreme versus moderate alcohol use was greater at younger ages, and approached one as age increased towards 90 years. This result reflects the comparatively low incidence of death through middle age. Consideration of the absolute risk of death, however, suggests that moderate drinkers have a substantial cumulative survival advantage over extreme drinkers throughout the adult lifespan. It has been suggested that wine drinking could be more favourably associated than other alcoholic beverages to the risk of CHD and some cancers. ${ }^{29-32}$ In this study beer use displayed more apparent risk patterns than wine consumption, particularly in men. Although we believe that this finding is relevant, we call for cautious interpretations of these results, as the lifestyle profile of wine and beer drinkers is profoundly different. The associations 
Women
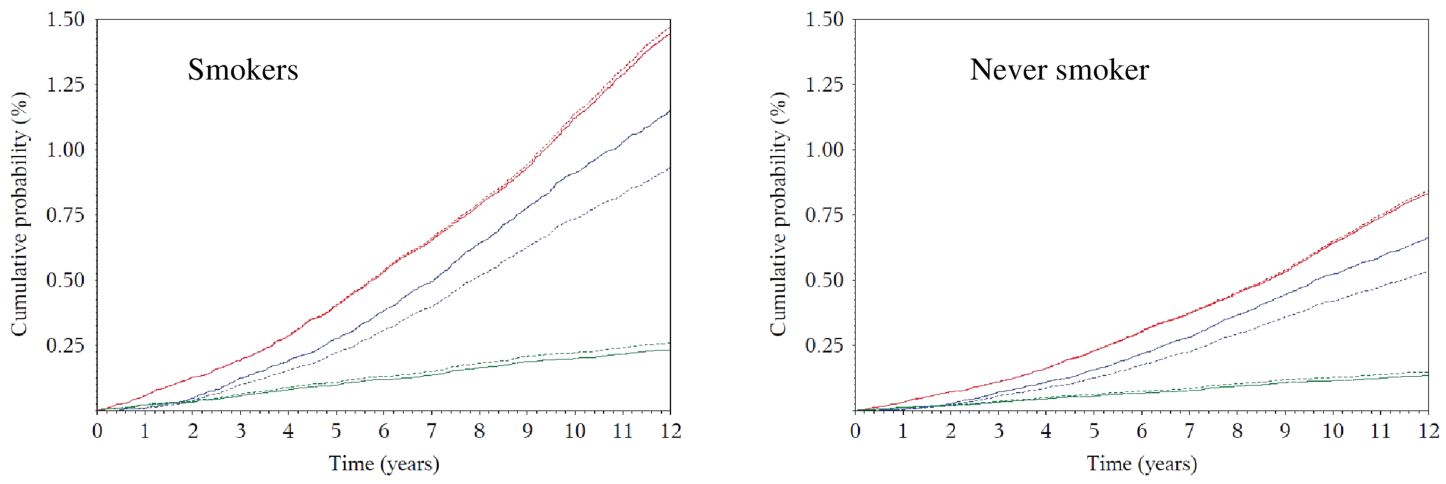

Men
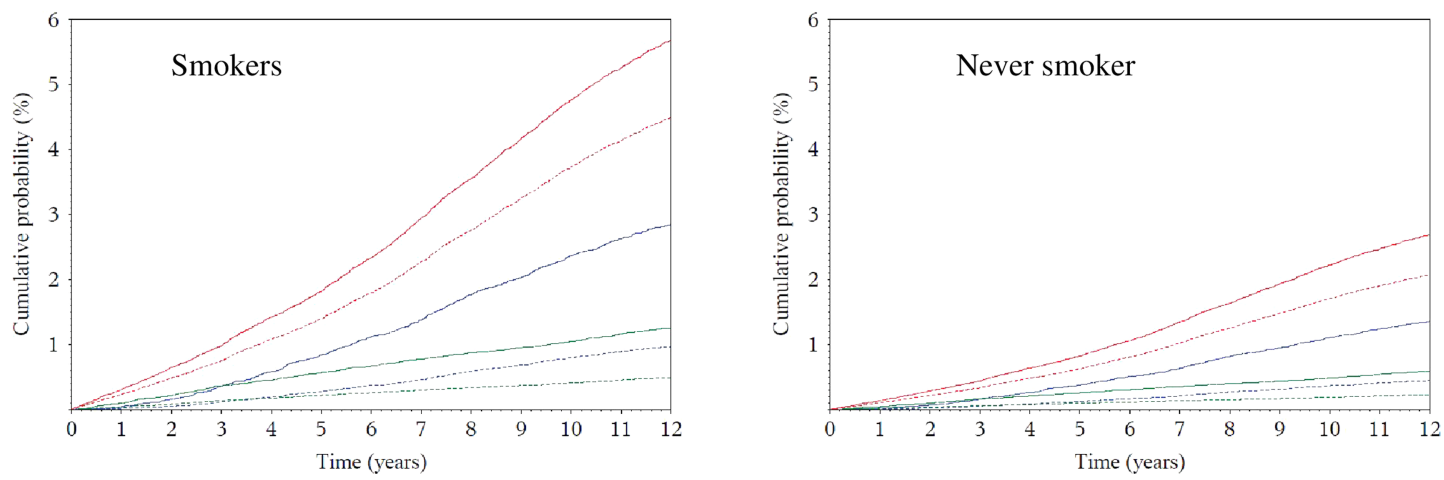

Figure 3 In competing risks analyses, sex-specific plots displaying cumulative probabilities of death due to CVD/CHD (red), alcohol-related cancers (blue) and violent death and injuries (green), for study participants aged 60 years according to heavy (=30 $\mathrm{g} /$ day in women and $=60 \mathrm{~g} /$ day in men, continuous line) and moderate $(0.1-4.9 \mathrm{~g} /$ day, dotted lines) lifetime alcohol use in current and never smokers in the EPIC study.

between alcohol and mortality were heterogeneous across countries in men, but not in women. This could be due to the larger amount of alcohol consumed in men than in women, naturally increasing the variability of exposure and the statistical power to detect associations, and of the larger variability characterising drinking habits in men, such as binge drinking, drinking during meals and other societal aspects. Although no

Table 4 Sex-specific estimates of rate advancement period (RAP) and associated $95 \% \mathrm{Cl}$ for overall and mortality due to ARCs, CVD/CHD and injuries and violent deaths, related to two scenarios of lifetime alcohol use. RAP estimates express the impact of a given exposure on the risk of death, by determining the time (in years) by which the risk of death is anticipated for study participants exposed, for example, all drinkers more than the threshold ( 5 or $15 \mathrm{~g} /$ day in Scenarios I and II, respectively), compared to non-exposed, that is, individuals drinking between $0.1 \mathrm{~g} /$ day and the threshold ${ }^{*}$

\begin{tabular}{|c|c|c|c|c|}
\hline & \multicolumn{2}{|l|}{ Scenario I } & \multicolumn{2}{|l|}{ Scenario II } \\
\hline & \multicolumn{2}{|c|}{ Threshold 5 g/day } & \multicolumn{2}{|c|}{ Threshold $15 \mathrm{~g} /$ day } \\
\hline & RAP (years) & $95 \% \mathrm{Cl}$ & RAP (years) & $95 \% \mathrm{Cl}$ \\
\hline \multicolumn{5}{|l|}{ Women } \\
\hline Overall & 0.36 & -0.05 to 0.76 & 0.83 & 0.26 to 1.39 \\
\hline CVD/CHD & 0.23 & -0.46 to 0.92 & 0.08 & -0.96 to 1.14 \\
\hline Alcohol-related cancers & 1.28 & -0.86 to 3.41 & 1.90 & -1.00 to 4.81 \\
\hline Injuries and violent deaths & -2.69 & -6.85 to 1.47 & -0.20 & -5.85 to 5.46 \\
\hline \multicolumn{5}{|l|}{ Men } \\
\hline Overall & 0.15 & -0.48 to 0.76 & 1.42 & 0.96 to 1.89 \\
\hline CVD/CHD & -0.53 & -1.57 to 0.50 & -0.01 & -0.82 to 0.81 \\
\hline Alcohol-related cancers & 2.59 & -0.30 to 5.49 & 5.03 & 3.07 to 7.00 \\
\hline Injuries and violent deaths & 7.59 & -2.82 to 18.02 & 11.83 & 3.92 to 18.17 \\
\hline
\end{tabular}


heterogeneity was observed by smoking status, mortality among smokers was higher than the mortality among non-smokers, so the absolute increase in risk associated with alcohol intake is more extreme among smokers. This differential increase in cumulative probability of death emphasises the central role of tobacco as a risk factor for mortality, as well as the potential extra harm of increased alcohol consumption.

In the EPIC study, although associations with alcohol were mostly apparent for ARC and violent death and injuries, absolute risks were highest for CVD/CHD in men, while CVD/CHD and ARC risks were of similar magnitude in women. In general, as individuals reporting a prevalent condition at recruitment (either cancer, diabetes, heart attack or stroke) were excluded from the analysis in an effort to minimise reverse causality, our estimates of absolute risks of death are possibly underestimated. RAP values were estimated to appreciate the risk benefit of alcohol drinkers if they were to modify their exposure, according to a counterfactual scenario. $^{21}{ }^{33}$ Consistently in men and women, RAP values for overall mortality were larger when the reference category was set to $0.1-15 \mathrm{~g} /$ day than when using a threshold of $5 \mathrm{~g} /$ day, thus indicating that, based on the EPIC study, the benefit for drinkers could be largest if their intake is reduced to levels below $15 \mathrm{~g}$ /day. These results are in line with findings of a recent work in the UK population, where the reduction of overall mortality was estimated to be optimised for alcohol reduction up to a median population level of $5 \mathrm{~g}$ /day. ${ }^{34}$

In a large prospective study in Europe, lifetime alcohol intake was significantly associated with overall and ARC-specific mortality. In men, positive associations were observed for violent deaths and injuries, while CVD and CHD deaths were not associated with alcohol use among drinkers. Our findings suggest that these associations do not differ between never and current smokers, and were stronger for beer than for wine drinkers.

\footnotetext{
Author affiliations

${ }^{1}$ International Agency for Research on Cancer, Lyon, France

${ }^{2}$ Department of Biostatistics, University of Copenhagen, Copenhagen, Denmark

${ }^{3}$ Potsdam-Rehbrücke Department of Epidemiology, German Institute of Human Nutrition, Nuthetal, Germany

${ }^{4}$ Department of Community Medicine, Faculty of Health Sciences, University of Tromso, The Arctic University of Norway, Tromsø, Norway

${ }^{5}$ Department of Research, Cancer Registry of Norway, Oslo, Norway

${ }^{6}$ Department of Medical Epidemiology and Biostatistics, Karolinska Institutet, Stockholm, Sweden

${ }^{7}$ Samfundet Folkhälsan, Helsinki, Finland

${ }^{8}$ Inserm, Centre for research in Epidemiology and Population Health (CESP), U1018, Nutrition, Hormones and Women's Health team, Villejuif, France

${ }^{9}$ Université Paris Sud, UMRS 1018, Villejuif, France

${ }^{10}$ IGR, Villejuif, France

${ }^{11}$ Cancer Epidemiology Unit, Nuffield Department of Population Health, University of Oxford, Oxford, UK

${ }^{12}$ Department of Public Health and Primary Care, University of Cambridge Addenbrooke's Hospital, Cambridge, UK

${ }^{13}$ Medical Research Council Epidemiology Unit, Institute of Metabolic Science, Addenbrooke's Hospital, Cambridge, UK
}

${ }^{14}$ Unit of Nutrition, Cancer Epidemiology Research Program, Environment and Cancer, Bellvitge Biomedical Research Institute (IDIBELL), Catalan Institute of Oncology (ICO), Barcelona, Spain

${ }^{15}$ Navarre Public Health Institute, Pamplona, Spain

${ }^{16}$ Consortium for Biomedical Research in Epidemiology and Public Health (CIBER Epidemiología y Salud Pública-CIBERESP), Murcia, Spain

${ }^{17}$ Escuela Andaluza de Salud Pública, Instituto de Investigación Biosanitaria de Granada, Granada, Spain

${ }^{18}$ CIBER Epidemiología y Salud Pública (CIBERESP), Spain

${ }^{19}$ Department of Epidemiology, Murcia Regional Health Council, Murcia, Spain

${ }^{20}$ Department of Health and Social Sciences, Universidad de Murcia, Murcia,

Spain

${ }^{21}$ Public Health Division of Gipuzkoa, Instituto BIO-Donostia, Basque

Government, Spain

${ }^{22}$ Nutrition Epidemiology Research Group, Department of Clinical Sciences,

Lund University, Malmö, Sweden

${ }^{23}$ Danish Cancer Society Research Center, Copenhagen, Denmark

${ }^{24}$ Department of Hygiene, Epidemiology and Medical Statistics, WHO

Collaborating Center for Food and Nutrition Policies, University of Athens

Medical School, Athens, Greece

${ }^{25}$ Hellenic Health Foundation, Athens, Greece

${ }^{26}$ Department of Epidemiology, Harvard School of Public Health, Boston,

Massachusetts, USA

${ }^{27}$ Bureau of Epidemiologic Research, Academy of Athens, Athens, Greece

${ }^{28}$ Cancer Registry and Histopathology Unit, "Civic-MP Arezzo" Hospital, ASP

Ragusa, Italy

${ }^{29}$ Epidemiology and Prevention Unit, Fondazione IRCCS Istituto Nazionale dei

Tumori, Milan, Italy

${ }^{30}$ Unit of Cancer Epidemiology, AO Citta' della Salute e della Scienza-

University of Turin and Center for Cancer Prevention (CPO-Piemonte), Turin,

Italy

${ }^{31}$ Human Genetics Foundation (HuGeF), Turin, Italy

${ }^{32}$ Molecular and Nutritional Epidemiology Unit, Cancer Research and

Prevention Institute-ISPO, Florence, Italy

${ }^{33}$ Department of Cancer Epidemiology, German Cancer Research Centre,

Heidelberg, Germany

${ }^{34}$ Department of Epidemiology, Julius Center for Health Sciences and Primary

Care, University Medical Center, Utrecht, The Netherlands

${ }^{35}$ Department of Statistics, Federal University of Rio Grande do Sul, Porto Alegre, Brazil

${ }^{36}$ Department of Epidemiology \& Biostatistics, School of Public Health,

Imperial College London, London, UK

${ }^{37}$ Department of Public Health, Section for Epidemiology, Aarhus University, Aarhus, Denmark

${ }^{38}$ Department of Cardiology, Aalborg University Hospital, Aalborg, Denmark

Contributors PF, IL, IR, PB and HB conceptualised the study and defined the analytical strategy. PF, IL, DCM, PKA and LN performed statistical analyses and provided preliminary interpretation of findings. PF, DCM, MJ, IL and PB contributed by drafting the manuscript. gEW, LD, LAD, GF, KEB, K-TK, EJD, $A B$, EM-M, CNS, LA, PW, AT, AO, ATR, VB, DT, RT, CA, CS, DP, KLI, RK, PP, JWJB, MG, TN, KO, ER, PB and IR played a key role in the acquisition of the data, and were active in searching for funding to continue the study. With respect to this work, they all critically helped in the interpretation of results, revised the manuscript and provided relevant intellectual input.

Funding This work was supported by the Direction Générale de la Santé (French Ministry of Health) (Grant GR-IARC-2003-09-12-01), by the European Commission (Directorate General for Health and Consumer Affairs) and the International Agency for Research on Cancer. The national cohorts are supported by the Danish Cancer Society (Denmark); the Ligue Contre le Cancer, the Institut Gustave Roussy, the Mutuelle Générale de l'Education Nationale and the Institut National de la Santé et de la Recherche Médicale (France); the Deutsche Krebshilfe, the Deutsches Krebsforschungszentrum, and the Federal Ministry of Education and Research (Germany); the Hellenic Health Foundation, the Stavros Niarchos Foundation and the Hellenic Ministry of Health and Social Solidarity (Greece); the Italian Association for Research on Cancer and the National Research Council (Italy); the Dutch Ministry of Public Health, Welfare and Sports, the Netherlands Cancer Registry, LK 
Research Funds, Dutch Prevention Funds, the Dutch Zorg Onderzoek Nederland, the World Cancer Research Fund and Statistics Netherlands (the Netherlands); European Research Council-2009-AdG 232997 and the Nordforsk, Nordic Centre of Excellence programme on Food, Nutrition and Health (Norway); the Health Research Fund, Regional Governments of Andalucýa, Asturias, Basque Country, Murcia (project 6236) and Navarra, Instituto de Salud Carlos III, Redes de Investigacion Cooperativa (RD06/0020) (Spain); the Swedish Cancer Society, the Swedish Scientific Council and the Regional Government of Skåne (Sweden); Cancer Research UK, the Medical Research Council, the Stroke Association, the British Heart Foundation, the Department of Health, the Food Standards Agency, and the Wellcome Trust (UK). The work undertaken by David C Muller was done during the tenure of an IARC, Australia postdoctoral fellowship, supported by the Cancer Council Australia.

Competing interests KEB reports grants from Cancer Research UK, during the conduct of the study. The rest of the authors declared no support from any organisation for the submitted work, no financial relationships with any organisations that might have an interest in the submitted work in the previous 3 years, no other relationships or activities that could appear to have influenced the submitted work.

Patient consent Obtained.

Ethics approval The study was approved by the Ethical Review Board of the International Agency for Research on Cancer, and by the local Ethics Committees in the participating centres.

Provenance and peer review Not commissioned; externally peer reviewed.

Data sharing statement Statistical code is available from the corresponding author by emailing ferrarip@iarc.fr.

Open Access This is an Open Access article distributed in accordance with the terms of the Creative Commons Attribution (CC BY 3.0) license, which permits others to distribute, remix, adapt and build upon this work, for commercial use, provided the original work is properly cited. See: http:// creativecommons.org/licenses/by/3.0/

\section{REFERENCES}

1. Thun MJ, Peto R, Lopez AD, et al. Alcohol consumption and mortality among middle-aged and elderly US adults. $N$ Engl J Med 1997;337:1705-14.

2. Bagnardi V, Blangiardo $M$, La Vecchia $C$, et al. A meta-analysis of alcohol drinking and cancer risk. Br J Cancer 2001;85:1700-5.

3. Rehm J, Baliunas D, Borges GL, et al. The relation between different dimensions of alcohol consumption and burden of disease: an overview. Addiction 2010;105:817-43.

4. Corrao G, Bagnardi V, Zambon A, et al. A meta-analysis of alcohol consumption and the risk of 15 diseases. Prev Med 2004;38:613-19.

5. Ronksley PE, Brien SE, Turner BJ, et al. Association of alcohol consumption with selected cardiovascular disease outcomes: a systematic review and meta-analysis. BMJ 2011;342:d671.

6. IARC. Alcohol consumption and ethyl carbamate. 96th edn. IARC; 2010.

7. WCRF/AICR. Food, nutrition, physical activity and the prevention of cancer: a global perspective. Washington, DC: AICR, 2007.

8. Rehm J, Mathers C, Popova S, et al. Global burden of disease and injury and economic cost attributable to alcohol use and alcohol-use disorders. Lancet 2009;373:2223-33.

9. Lim SS, Vos T, Flaxman AD, et al. A comparative risk assessment of burden of disease and injury attributable to 67 risk factors and risk factor clusters in 21 regions, 1990-2010: a systematic analysis for the Global Burden of Disease Study 2010. Lancet 2012;380:2224-60.

10. Bergmann MM, Rehm J, Klipstein-Grobusch K, et al. The association of pattern of lifetime alcohol use and cause of death in the European Prospective Investigation into Cancer and Nutrition (EPIC) study. Int J Epidemiol 2013;42:1772-90.

11. Riboli E, Hunt KJ, Slimani N, et al. European Prospective Investigation into Cancer and Nutrition (EPIC): study populations and data collection. Public Health Nutr 2002;5:1113-24.

12. Ferrari $P$, Jenab $M$, Norat $T$, et al. Lifetime and baseline alcohol intake and risk of colon and rectal cancers in the European Prospective Investigation into Cancer and Nutrition (EPIC). Int $J$ Cancer 2007;121:2065-72.

13. Ferrari P, Day NE, Boshuizen $\mathrm{HC}$, et al. The evaluation of the diet/ disease relation in the EPIC study: considerations for the calibration and the disease models. Int $J$ Epidemiol 2008;37:368-78.

14. Royston P, Parmar MK. The use of restricted mean survival time to estimate the treatment effect in randomized clinical trials when the proportional hazards assumption is in doubt. Stat Med 2011;30:2409-21.

15. Heinzl H, Kaider A. Gaining more flexibility in Cox proportional hazards regression models with cubic spline functions. Comput Methods Programs Biomed 1997;54:201-8.

16. Sauerbrei $W$, Royston $P$. Building multivariable prognostic and diagnostic models: transformation of the predictors by using fractional polynomials. J R Stat Soc A 1999;162:71-94.

17. Royston $P$, Ambler $G$, Sauerbrei W. The use of fractional polynomials to model continuous risk variables in epidemiology. Int $J$ Epidemiol 1999;28:964-74.

18. Andersen PK, Geskus RB, de WT, et al. Competing risks in epidemiology: possibilities and pitfalls. Int J Epidemiol 2012;41:861-70.

19. Putter H, Fiocco M, Geskus RB. Tutorial in biostatistics: competing risks and multi-state models. Stat Med 2007;26:2389-430.

20. Rosthoj S, Andersen PK, Abildstrom SZ. SAS macros for estimation of the cumulative incidence functions based on a Cox regression model for competing risks survival data. Comput Methods Programs Biomed 2004;74:69-75.

21. Brenner H, Gefeller O, Greenland S. Risk and rate advancement periods as measures of exposure impact on the occurrence of chronic diseases. Epidemiology 1993;4:229-36.

22. SAS Institute Inc., Cary, NC, USA, 2002. http://www.stat.ncsu.edu/ research/sas/faq/citation.html

23. Stata Statistical Software: Release 12. VersionStataCorp. College Station, TX: StataCorp LP., 2011.

24. Zaridze D, Lewington S, Boroda A, et al. Alcohol and mortality in Russia: prospective observational study of 151000 adults. Lancet 2014;383:1465-73.

25. Thun MJ, Peto R, Lopez AD, et al. Alcohol consumption and mortality among middle-aged and elderly US adults. $N$ Engl J Med 1997;337:1705-14.

26. Lin Y, Kikuchi S, Tamakoshi A, et al. Alcohol consumption and mortality among middle-aged and elderly Japanese men and women. Ann Epidemiol 2005;15:590-7.

27. Fillmore KM, Stockwell T, Chikritzhs T, et al. Moderate alcohol use and reduced mortality risk: systematic error in prospective studies and new hypotheses. Ann Epidemiol 2007;17(5 Suppl):S16-23.

28. Naimi TS, Brown DW, Brewer RD, et al. Cardiovascular risk factors and confounders among nondrinking and moderate-drinking US adults. Am J Prev Med 2005;28:369-73.

29. Klatsky AL, Friedman GD, Armstrong MA, et al. Wine, liquor, beer and mortality. Am J Epidemiol 2003;158:585-95.

30. Maskarinec G, Meng L, Kolonel LN. Alcohol intake, body weight, and mortality in a multiethnic prospective cohort. Epidemiology 1998;9:654-61.

31. Gronbaek M, Becker U, Johansen D, et al. Type of alcohol consumed and mortality from all causes, coronary heart disease, and cancer. Ann Intern Med 2000;133:411-19.

32. Renaud SC, Gueguen R, Siest G, et al. Wine, beer, and mortality in middle-aged men from eastern France. Arch Intern Med 1999;159:1865-70.

33. Gellert C, Schottker B, Holleczek B, et al. Using rate advancement periods for communicating the benefits of quitting smoking to older smokers. Tob Control 2013;22:227-30.

34. Nichols M, Scarborough $\mathrm{P}$, Allender S, et al. What is the optimal level of population alcohol consumption for chronic disease prevention in England? Modelling the impact of changes in average consumption levels. BMJ Open 2012;2:e000957. 